\title{
AdvanCES In Automatic InSECt Classification
}

\author{
Siti N. A. Hassan, Nadiah S. A. Rahman, Zaw Zaw Htike and Shoon Lei Win \\ Faculty of Engineering, IIUM, Kuala Lumpur, Malaysia
}

\begin{abstract}
Entomology has been deeply rooted in various cultures since prehistoric times for the purpose of agriculture. Nowadays, many scientists are interested in the field of biodiversity in order to maintain the diversity of species within our ecosystem. Out of 1.3 million known species on this earth, insects account for more than two thirds of these known species. Since 400 million years ago, there have been various kinds of interactions between humans and insects. There have been several attempts to create a method to perform insect identification accurately. Great knowledge and experience on entomology are required for accurate insect identification. Automation of insect identification is required because there is a shortage of skilled entomologists. This paper provides a review of the past literature in vision-based insect recognition and classifications. Over the past decades, automatic insect recognition and classification has been given extra attention especially in term of crop pest and disease control. This paper details advances in insect recognition, discussing representative works from different types of method and classifiers algorithm. Among the method used in the previous research includes color histogram, edge detection and feature extraction (SIFT vector). We provides discussion on the state-of-the-art and provides perspective on future research direction in insect recognition and classification problem.
\end{abstract}

\section{KEYWORDS}

Vision-based Entomology, Color Features, Shape Features, Machine Learning

\section{INTRODUCTION}

Recently, image-based insect recognition has emerged as a new field of study concerning image processing and intelligent pattern recognition replacing inefficient traditional technique [1]. Requests for insect recognition and classification to be carried out more efficiently have become pressing. In response, this image-based technology is used to improve the shortcomings of the traditional method such as manual identification of insects by the experts as well enhancing accuracy and saving time. In fact, many research have been done to develop this technology to become one of the most crucial method in classification and recognition of insects.

There have been many successful attempts of using machine learning in automation of labour intensive tasks [14-30]. Image-based insect recognition has wide range of applications especially in agriculture, ecology and environmental science [5]. It generally can be utilized in prevention of plant disease and insect pests, plant quarantine and as an essential part of eco-informatics research. Insect detection has to be taken into a serious measure as insect presents an especially severe threat because they can multiple alarmingly in a short period of time [12]. This new intelligent system is very beneficial especially to laymen who do not possessed professional knowledge in distinguishing many species of insects.

Initially, thresholding seems to be the best approach for insect detection and classification which appear dark relative to the light brown color of the grain. However, early tests shows that it can be many false alarm resulting from chaff and other permitted admixture such as rapeseeds and even the shadows between [12]. Considering this issue, researchers come out with many other method for detecting objects in real time specifically insects in grains. 


\section{Characteristics of InSECTS}

Most of the insects are composed of several sub parts which are antennae, tails, wings and etc [5]. These parts are extracted in image processing method to be used in recognition of insects as well as other important features such as color and shape. Majority of the researchers use wing as the most crucial part for feature extraction in classification of an insect. In order to develop high performance and feasible solution in insect classification and recognition, the research starts at the beginning of 1990s where insect math morphology is combined with computer technology [1]. Although classification of insect is determined by morphological and autecological characteristics, but it is unattainable to analyze the autecological features through computer analysis [3].

\section{PRinCiPles Of IMAge-BASED ReCOGNition}

The general principles of image-based recognition and classification are image acquisition and image processing. Image acquisition is done by selecting a number of different species of insects as the specimen of the research. A picture of each species selected is taken using any camera to obtain an image. All the images are acquired and saved.

The next stage is image preprocessing stage where the image obtained will undergo several process. The image in color scale will be converted to grayscale. Next, the grayscale image will be converted ti binary image where only white and black color is produced. The region of interest (ROI) will be separated from the background image. The next step is image processing. The image will undergo feature extraction, and the features extracted will undergo classification process. At this stage, different method will produce different output. There are several methods used for insect recognition such as fuzzy classifier, nearest neighbor classifier and also artificial neural network (ANN) [4]. For example, some of the researchers used template matching and some used color histogram technique. As the world is developing, researchers come out with novel method in recognizing different type of insects to improve the accuracy in real time. Further details on the approaches and method used in classification of insects will be discussed in the next section.

\section{STATE OF THE ART}

\subsection{Colour Histogram and GLCM}

Zhu Le Qing and Zhang Zhen[1] introduced their research in using color histogram and Gray Level Co-occurrence Matrix (GLCM) for insect recognition. Image preprocessing algorithm is used to segment out the region of interest (ROI) from the insect image. Then, color features which are represented by color histogram are extracted from ROI that can be used for coarse color matching. The matching is done by comparing the correlation of the feature vectors with certain threshold [1]. The image samples that passed this stage will undergo the next stage that is fine level matching[1]. The fine level features are represented by coefficients such as energy, entropy and correlation of GLCM of preprocessed image blocks.

In the research, one hundred species of insects are selected as samples. All the image samples are captured from the right wing of the insects. A part of them are forewing with backwing, the rest are forewing. The image is resized to speed up the processing speed and then filtered using mean shift algorithm [2]. Then, it is converted to gray scale image and binarized with certain threshold. To reduce the effect of rotation and tranlation during image acquisition, a universal coordinate must be located by connecting the centroid of the foreground region and rotate the image around 
the centroid until the connected line is horizontal [1]. Least-square method is applied to fit the upedge and downedge of the insect wing with straight lines. ROI is determined afterwards and the image is aligned using Gaussian before feature extraction[1].
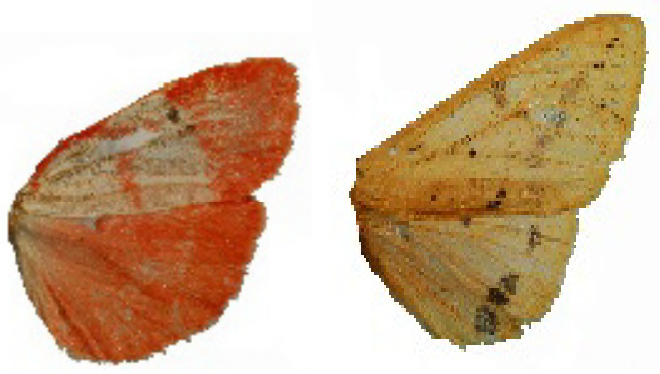

Figure 1: Forewing together with backwing of the acquired image [Adapted from Zhu\&Zhang, 2010]

The color image is transformed from RGB (Red-Green-Blue) space into HSV (Hue-SaturationValue) space before construct the histogram. To minimize the effect of illumination variation, only hue and saturation is take into consideration[1]. The histogram for hue and saturation component is calculated and it shows that the histogram for same species is distributed similarly.

As for GLCM, it estimates image properties related to second order statistics [20] for example energy, entropy, correlation and homogeneity[1]. This technique can identify insects from low resolution wing images and the efficiency and accuracy is proven[1].

(a)

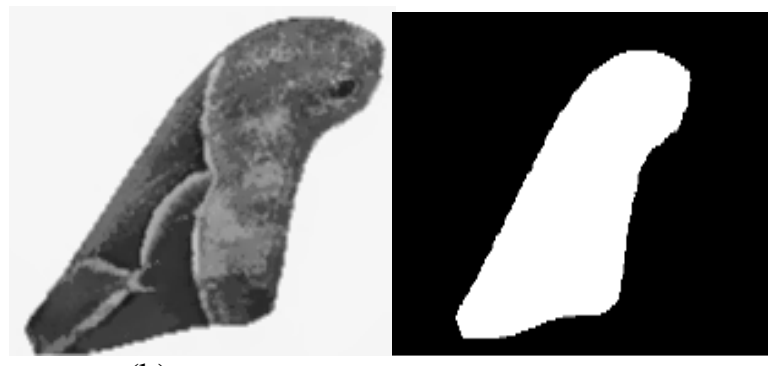

(b)

Figure 2: (a) Gray scale image (b) Binary image [Adapted from Zhu\&Zhang, 2010]

\subsection{Pattern Recognition}

According to Kim, Lim, Cho and Nam (2006), this research is focusing in identify butterflies and ladybugs. The image of the insects are acquired and noise rejection is processed using color information method. Edge detection technique is applied to RGB space after the pre-processing by top-hat filtering with certain threshold. The detected edge line is analyzed by chain code analysis. To improve the end result, the processed image undergo max/min filtering. 


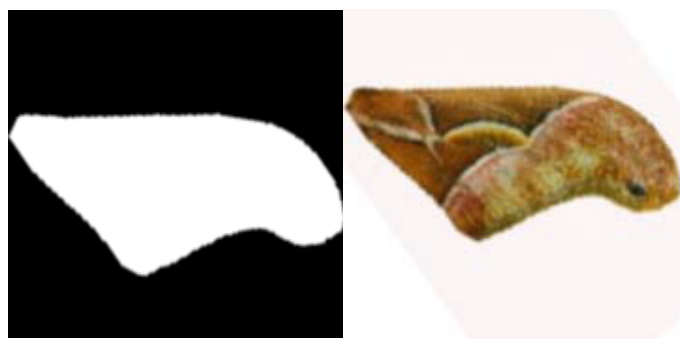

Figure 3 : Region of Interest and Aligned color image [Adapted from Zhu\&Zhang, 2010]

The computer will process the data and the program counts the butterfly and ladybug in a picture that is included with many kinds of insect. By using this method, the researchers claimed that they attained recognition about $90 \%$ as a whole [3].

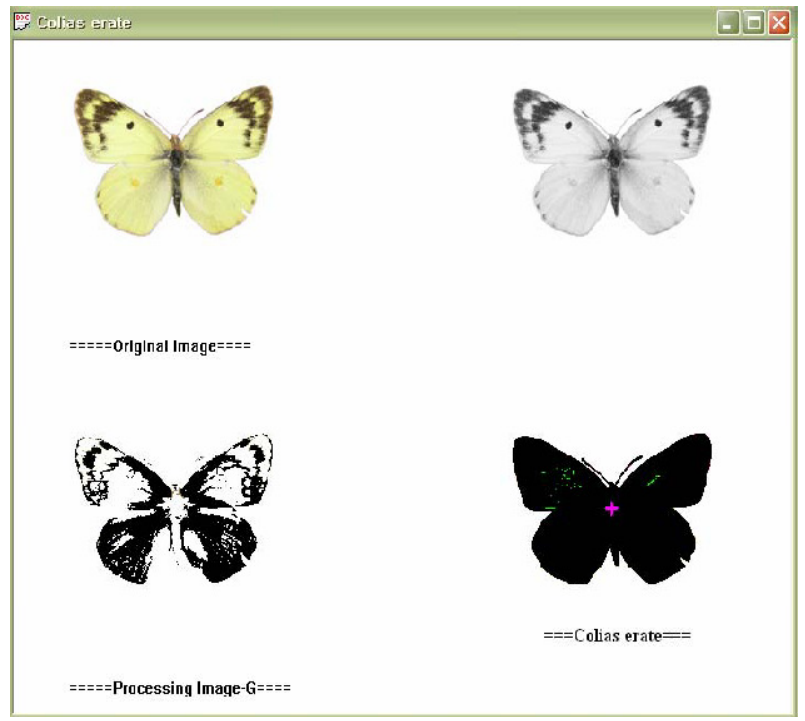

Figure 4: The program of insect discrimination [Adapted from Lim, Cho, Nam\&Kim,2006]

Another similar research was done by the Yang et al [7] where the process of insect identification is focused on pattern recognition technology. Pattern recognition can be specifically defined as "the act of taking in raw data and taking an action based on the category of the pattern" [7]. They further explained that the process involve three stages which are, input data and information collected from the sample using sensor, feature extraction mechanism that compute numeric or symbolic data from the sample, and classification scheme that determine the group of the sample[7]. Meanwhile, image segmentation is defined as the process on which the image is segregate into several portions where each portions grouped together pieces that share similar characteristic such as intensity or texture in order to generate non-overlapping image [7].

For success in insect recognition, they had collected several features of insect consists of rectangularity, elongation, roundness, eccentricity, sphericity, lobation, compactness and seven Hu moment invariants[7]. There are three classes of image features i.e colour, texture and edges. However, edge extraction seems to be the best approach since it allowed minimal interference of light and noise[7]. This type of process employed the procedure of machine learning to train the algorithm to match with the image loaded and the result were saved in database. There are variety of classifiers that offer different approaches to sort an image. And each classifiers were tested on the samples to determine the best classifier that can successfully fit the data into respective group. 
In this study, the researchers claimed that Random trees algorithm is the best choice since it surpassed other classifiers in the test of insects' image data samples [7].

The flowchart in Figure 5 shows the summary of training and recognition process involve in insect's classification. The solid line represent the flow of data signifies the process of training the classifiers whereas the dotted line signifies the path taken by a new loaded image to test the corresponding classifier.

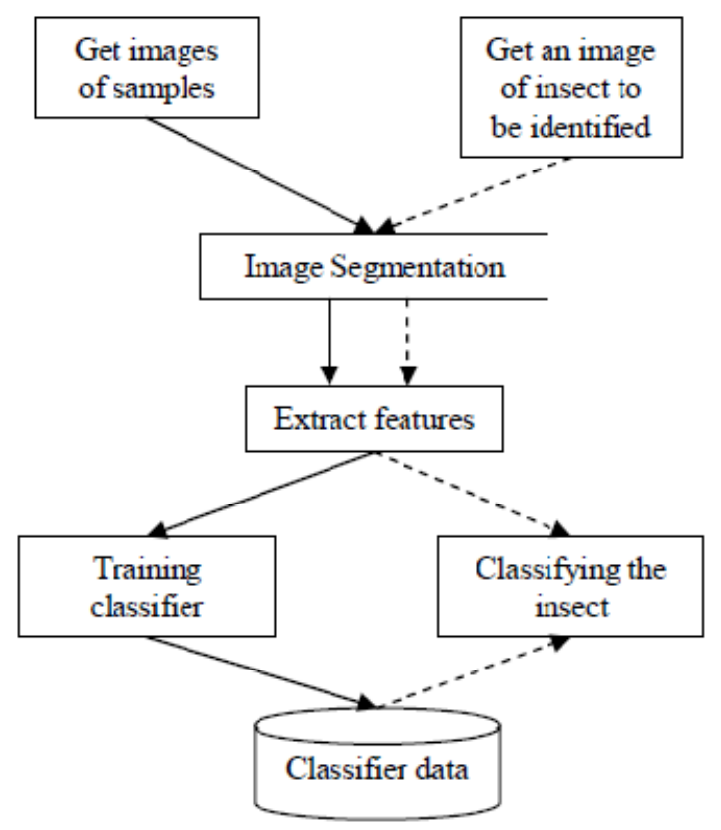

Figure 5: Main Flowchart of Training and Recognition [Adapted from Yang et al, 2010]

For image segmentation, they have been exercising a seven steps for classifying an insect. The seven procedures are: First, the image is loaded from a file. Then, image is converted to grayscale. Third, the edged is extracted using Canny edge algorithm. Close operator is used on the edges image. The next step would be finding an image contour by using contour algorithm. The biggest contour shows the contour of the insect that previously been loaded.

Contour feature extraction benefitted in the processing image with different image sizes an angles. However, the disadvantage would be obvious if the species have similar contour since the system cannot differentiate those contours. Hence, the solution to this problem is by exploiting image color and texture.

\section{EXTENSION THEORY}

Extension theory is a method that constructed the matter element matrix of the insect based on mean and variance of the image features [4]. The matter-element matrix to be recognized includes the object itself, the feature and the feature value of the object in this case is an insect. The first step to be done in extension method for classification of insects is feature extraction of insects [4]. The image will be captured in real time and converted to gray scale image, segmented by the adaptive method so that the binary insect image is separated from the grain and the background. Seventeen morphological features are extracted from the binary image and then are normalized [18]. Afterward, the matter element of features of the insects are constructed. According to " $3 \delta$ " 
property of the normal distribution, if the object belonged to a class, then each feature of the object should be within the threefold variance with $99.7 \%$ probability. Otherwise some features should be outside of the threefold variance [4].

The system is trained to identify the nine species of the insects given 135 samples. Classical field matter element and controlled field matter element matrix of each species is formed. The correlation degree is calculated [4]. The higher correlation degree between the object to be recognized and a class, it means that it is much closer to that class. The correlation degree was simple to calculate, easy to use, fast and efficient. The accuracy of the classifier is increased compared to fuzzy classifier and k nearest neighbor classifier [4].

\section{HYBRID APPROACH}

In this research, An Lu, Xinwen Hou, Cheng-Lin Liu and Xiaolin Chen try to use a hybrid approach in classifying insects called discriminative local soft coding (DLsoft) [5]. In soft coding strategies, a feature vector of the insect image is encoded by a kernel function of distance. As for the discriminative part, Multiple Kernel Learning (MKL) is used for the classification to improve the drawbacks of the soft coding alone.

This method is test on the fruit fly, Tephritidae that consists of different species. The dataset is divided into two; training set and testing set. Sample used for both dataset is an image of Tephritidae. First, all the images are transformed into gray scale. SIFT features of patches are extracted by densely sampled from each image. This is done so that each image will be represented by SIFT features. SIFT is done by dividing the images into several section of squares and each section will compute its own histogram. The local soft codes and discriminative is calculated. Subsequently, max pooling over all the vectors of patches in the same image is calculated. The final presentation of an image sample is spatial pyramid pooled vector which can be used by any machine learning method for classification [6].

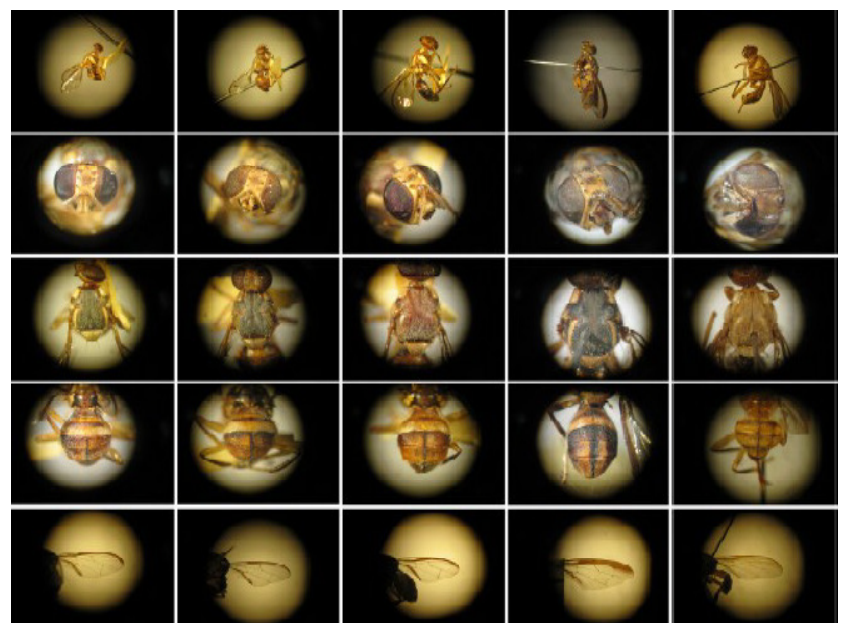

Figure 6: Example of our Tephritidae dataset: each column is corresponding to one species and the rows are respectively whole body, head, thorax, abdomen and wing photographs of the corresponding species taken by a microscope camera [Adapted from An Lu, Xinwen Hou, Cheng-Lin Liu \& Xiaolin Chen,2012) 


\section{STACKed SPatial-Pyramid KeRnel}

In another study, Larios et al. [8] shows some agreement with the previous study of Yang et al in terms of using Random Trees method as classifiers, however their study has added a new angle to improve the result. Larios et al [8] identified and described Stacked Spatial-Pyramid Kernel method to boost up the performance of standard stacking method hence provides greater accuracy for the sample classification.

Stacking is one of digital image processing technique that combines multiple classifiers with each classifiers to manage their own feature space. Since only the raw combination scores are being submitted as input to the final classifiers, the conventional stacking method has disadvantage of losing spatial information belonged to the features. In order to tackle the problem, Larios et al [8] introduced new stacking structure that has the capability to retain the spatial information as well as increase the classification result accuracy by quantizing input data. The authors believed that the method surpass other classifiers in grouping insects sample with variety of dissimilar feature types. In addition to that, the proposed method is capable to handle sample image with different 3D positions, orientations, and developmental and degradation stages with wide intra-class variation.

The study clarify three main steps of insect recognition process. First, the HOG and SIFT analysis are constructed from the edge and dense grid of patches provided by the image samples while beam angle histogram taking care of sample's shape. This three descriptors are significant in determine region of interest for local features. The appearance and shape features scores obtained from random trees method is exploited to draw the spatial histogram that shows relative position information. The whole process is illustrated as diagram in Figure 6.

This whole process are employed in the recognition and classification of species orders for water quality assessment. The species orders include three small organism namely, Ephemeroptera (mayflies), Plecoptera (stoneflies), and Trichoptera(caddisflies), often known as EPT [19]. These EPT have contrast capability from each other to induce water pollution thus, their accumulation providing a yardstick in water assessment quality.

Larios et al [8] stated that this method has the ability to combine dissimilar local features gathered by different detectors and descriptors yielding in a vast set of samples of large appearance variation as a result of assorted insect position and orientation, natural diversity, growing stage and degradation after the insect is captured. Next step is extracting useful region for local features. Commonly, shape, edge key points and patches managed to attain the required features. These features illustrated by HOG and SIFT descriptors while beam angle histogram explain the shape. The spatial histogram then displayed the relative position information built from combination of local classification scores previously calculated from random trees classifier. Spatial histogram is said to have the ability of maintaining information about the quantity of features identified in different image regions. 


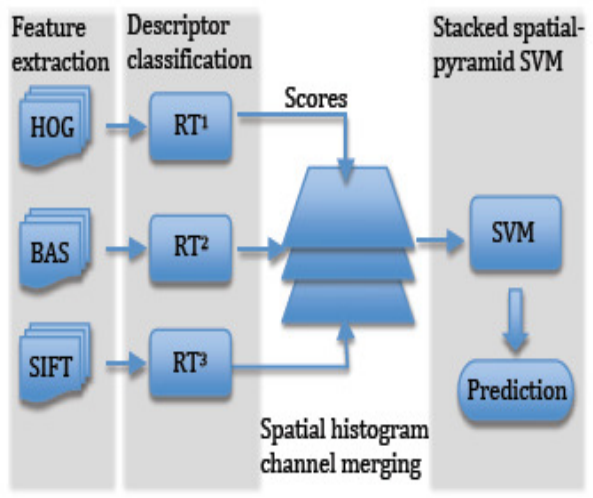

Figure 7: Overview of the stacking classification architecture. [Adapted from Larios et al, 2010]

In order to acquire the parameters for the stacked spatial pyramid kernel SVM learning algorithm, logarithmic grid search are performed with five folds cross validation. In this training set consist of multi-class experiments, one versus all frameworks were utilized. Larios et al [8] specified that "the spatial-pyramid kernel $\mathrm{K}$ is used in the loss and discriminant functions of the SVM learning. This kernel is well suited for the histogram image representation, because classification scores just like assignment counts, can be accumulated in the spatial pyramid."

\section{ONTOLOGY-BASED INSECT RECOGNITION}

On the other hand, Huang Shiguo et al. [9] proposed insect identification based on ontology framework. The authors claimed that ontology based image retrieval has gained much popularity in semantic image retrieval in recent years. This method is similar with other past researches in the beginning where the image features are extracted from the samples database. The features then being stored in feature database. Ontology based recognition system consist of a layer that hold four types of system identification namely, visual feature ontology, image feature ontology, classification module, and Insect Morphology and Taxonomy Ontology(IMTO)[9]. This layer will give support and assist the insect image recognition and annotation. Visual feature extract the image while classification module pair the image with the insect image previously stored in feature knowledge database. 


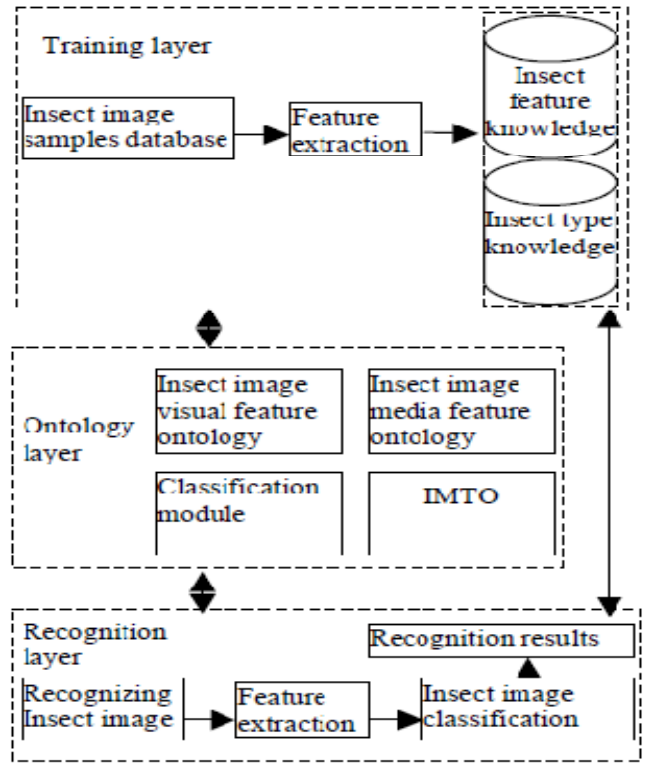

Figure 8: Ontology based insect recognition [Adapted from Huang et al, 2009]

Image visual ontology is a significant element in ontology based insect classification. In this stage, visual feature and their properties of insect is extracted from an image. The properties comprise of local features, feature subspace, texture features, and color features. These properties are expressed by the SIFT or SURF descriptors[9]. For IMTO layer, insects' information is build based on their family species until family tree is developed which consist of one head and several branches of family species. This is to recognize the insect's physical build to identify the similarities or dissimilarities between each family species. Image media feature ontology contribute in term of image retrieval background such as when and how the photo were taken, environment information and image storage format (JPEG, BMP etc). All of these layers communicates with each other to construct a relationship in recognizing and identified insect [9]. The diagram in Figure 7 shows the framework of ontology based insect recognition.

\section{KNN-SPECTRALREGRESSION LDA}

$\mathrm{Li}$ at al. [10] provides another alternative in insect recognition and classification field. According to $\mathrm{Li}$ at al $\mathrm{KNN}$-Spectral regression LDA is one of the best method that can be applied in automatic insect recognition. This method is based on the face recognition principle.

The summary of the procedures is represent in Figure 8. Raw sample image of an insect is loaded and be process to reduce the dimension by applying dimension reduction algorithm. Next step would be obtaining feature subspace from pre-process training sample[10]. On the same time, recognized image is projected into feature subspace. Therefore, the linear combination of feature subspace vector of unrecognized image is obtained by comparing from the sample image. By measuring the similarity between coefficient vector of unrecognized image and that of training samples, KNN method is employed to recognize insects[10]. 


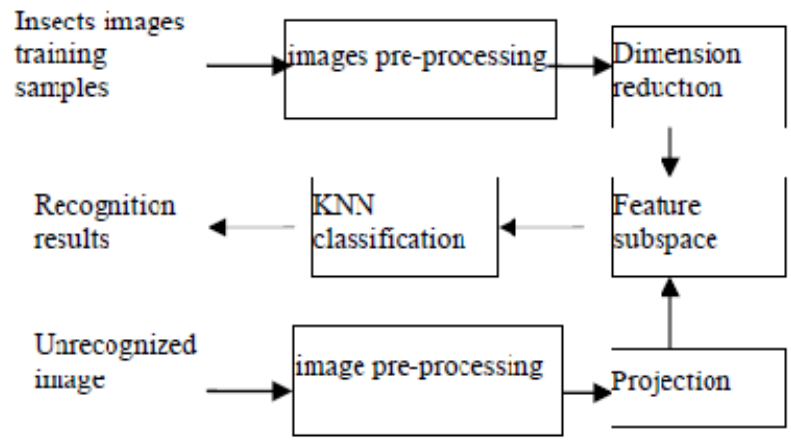

Figure 9: Dimension Reduction for insect recognition [Adapted from Li et al, 2009]

The function of LDA algorithm is to spread the distribution of different classes samples wider into space while the sample that belong to the same feature is grouped more closely to each other. In the other word, inter scatter are hold far apart from each other whereas intra scatter degree is kept small. The problem can arise if there is only one intra scatter which lead to undetermined best projection direction.

The coeficient from PCA and LDA are selected as input to KNN algorithm[10]. To categorize an unrecognized object, KNN algorithm is used. K-neighbours is used to compute the nearest sample to image class thus providing a more dense class sample to increase the accuracy of insect classification. This research confirmed that KNN-Spectral Regression LDA outdo the PCA and Run-length matrix with $90 \%$ recognition accuracy rather than $75 \%$ in PCA.
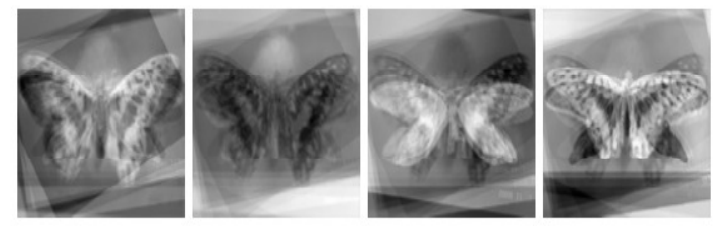

Figure 9: Insect Feature Subspace from PCA [Adapted from Li et al, 2009]
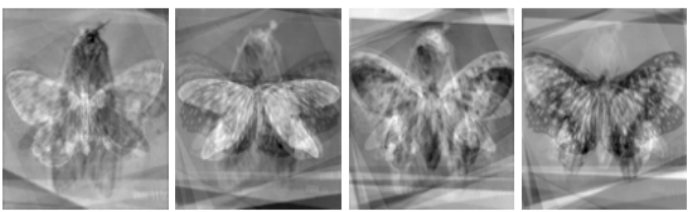

Figure 10: Insect feature subspace from spectral regression LDA [Adapted from Li et al, 2009]

\section{Histogram of Local Appearances Features}

Larios et al. [17] mentioned that the goal of the research is to develop general-purpose computer vision methods, and associated mechanical hardware, for rapid-throughput image capture, classification, and sorting of small arthropod specimens. The focus in this research is on stonefly (Plecoptera) larvae for the biomonitoring of freshwater stream health. Stream quality measurement could be significantly advanced if an economically practical method were available for monitoring insect populations in stream substrates [11,17].

Recognition of stonefly butterfly is challenging as they are highly interconnected, exhibit a high degree of various species in size and colour and some species are hard to differentiate visually 
despite prominent dorsal patterning [13]. To encounter this problem, the researchers come out with different approach called the bag-of-features approach. It extracts a bag of region-based features from the image without take into account their relative spatial arrangement [17].

The bag-of-features approach involves five stages which are region detection, region description, region classification, combination of detected features into a feature vector and final classification of the feature vector. For region detection, they are using three different operator that is Hessianaffine detector, Kadir entropy detector and principle curvature-based region detector (PCBR) [17]. The detected region is then described using SIFT representation [17]. In classification into features, a histogram is assigned to each feature formed consisting of the number of SIFT vectors [13].

In the fourth stage or phase, feature vector is created for each of the three region detectors using GMM (Gaussian Mixture Model). These features then formed the concatenated feature histogram $(\mathrm{CFH})$ method that allows the use of general classifier from the machine learning literature. Finally, the classification of the specimens is done by an assembler of logistic model tress [13]. This stage is to train a classifier to assign the correct labels to the bag of keywords where this keywords is obtained from the clustering of SIFT vectors. There are many ways to train the classifier and one simple method is to compute a histogram where the $i$-th element corresponds to the number of occurrences in the image of the $i$-th keyword. Another classification strategy is to employ distance-based learning algorithms such as the nearest-neighbor method. This involves defining a distance measure between two bags of keywords such as the minimum distance between all keywords from one bag and all keywords from the other bag [13,17].

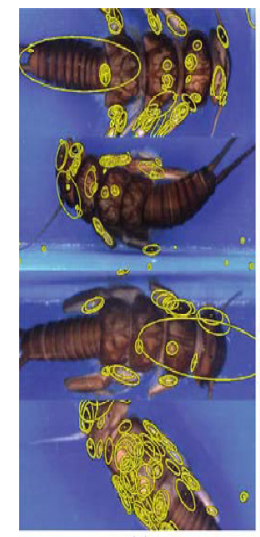

(a)

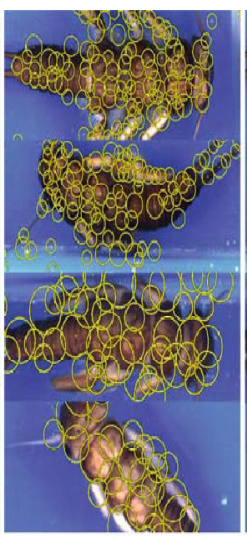

(b)

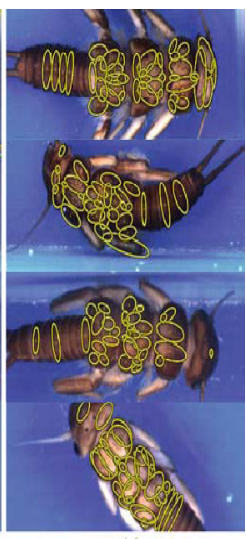

(c)

Figure 11: Visual Comparison of the regions output by the three detectors on three Calineuria specimens.

a) Hessian-affine, b) Kadir entropy, c) PCBR [Adapted from Larios et al., 2007]

\section{Challenges in InSect Classification}

There are several issues emerged in the pursue of insect recognition as stated by Kevin J. Gaston, et al (2004, cited in Yang, et al, 2010) [7] which he clearly pointed out that the species of insect are too complicated, too threatening, too different and the problem also lies in the finance factor. Adding to that, Yang et al claimed that for some species especially from the same genus are very difficult to be differentiate using only an image as parameter even the expert sometimes need to observe the insect's behavior or use other methods to identify them.

The other challenge that could be arise is the unfair distribution of samples per species. This situation could lead to different performance accuracy. 


\section{Limitations of THE State OF THE ART Systems}

There are several limitation and challenges that are encountered in the techniques used in the previous research. Some insects of the same species and type has different color histogram that make the hard to be classified. Especially when using color histogram to classify the insects. Other than that, since the body of the insects is covered by colourful scale, some features are hard to be extracted and to be computed the feature vectors. Hence, they need to be extracted by using additional information such as behaviour feature.

At some stage, the insect classification might become a little complex and where the system has to train so many type of insects as the insects consist of so wide range of species.

\section{Conclusion}

This paper review several methods used in machine vision learning in detecting and classifying insects based on their features, colors, shape and etc. Each method has the advantages and disadvantages in detecting insects. Among the method used, color histogram seems to be the best approach in classifying and recognizing species of insects. This is because the color histogram is using SIFT extraction feature to describe the image of the insect to be recognized. Each image acquired is divided into several square similar in size and each of the square of images has its own histogram. Even though the detected insects is not in the same position in the trained image, the system still can identified which type of insect based on the color histogram.

\section{REFERENCES}

[1] L.Q. Zhu, Z. Zhang, "Auto-classification of Insect Images Based on Color Histogram and GLCM", Seventh International Conference on Fuzzy Systems and Knowledge Discovery, 2010.

[2] D. Comaniciu, P. Meer, "Mean shift: a robust approach toward feature space analysis", IEEE Transactions on Pattern Analysis and Machine Intelligence, 24(5): pp.603-619, 2002.

[3] J. H. Lim, et al., "Development of a Classification Algorithm for Butterflies and Ladybugs", 2006.

[4] H.T. Zhang, et al., "Extension Theory for Classification of the Stored-Grain Insects", International Conference on Machine Vision and Human-machine Interface, 2010.

[5] A. Lu, et al., "Insect Species Recognition using Discriminative Local Soft Coding", 21st International Conference on Pattern Recognition, 2012.

[6] J. Yang, et al., "Linear spatial pyramid matching using sparse coding for image classification", Computer Vision and Pattern Recognition(CVPR), 2009.

[7] H. Yang, et al., "Research on Insect Identification Based on Pattern Recognition Technology", Sixth International Conference on Natural Computation, 2010.

[8] N. Larios, et al., "Stacked Spatial-Pyramid Kernel: An Object-Class Recognition Method to Combine Scores from Random Trees", 2010.

[9] S. G. Huang, et al., "Ontology-Based Insect Recognition”, 2009.

[10] L. Xiao-Lin, et al., "KNN-Spectral Regression LDA for Insect Recognition”, First Interbational Conference on Information Science and Engineering, 2009.

[11] W.L. Hilsenhoff, "Rapid field assessment of organic pollution with a family level biotic index",Journal of the North American Benthological Society, Vol. 7, pp.65-68, 1988.

[12] E.R. Davies, "Computer and Machine Vision:Theory, Algorithms, Practicalities",United States, Academic Press, Fourth Edition, 2012.

[13] N. Larios, et al., "Automated insect identification through concatenated histograms of local appearance features: feature vector generation and region detection for deformable objects", Machine Vision and Applications, 2008.

[14] Z.Z. Htike, S.L. Win "Recognition of Promoters in DNA Sequences Using Weightily Averaged Onedependence Estimators”, Procedia Computer Science, Volume 23, 2013, pp. 60-67, ISSN 1877-0509. 
[15] Z.Z. Htike, S.L. Win "Classification of Eukaryotic Splice-junction Genetic Sequences Using Averaged One-dependence Estimators with Subsumption Resolution", Procedia Computer Science, Volume 23, 2013, pp. 36-43, ISSN 1877-0509.

[16] Z.Z. Htike, et al., "A Monocular View-Invariant Fall Detection System for the Elderly in Assisted Home Environments", 7th International Conference on Intelligent Environments (IE), 2011, pp.40-46, 25-28 July 2011.

[17] N. Larios, el al., "Automated Insect Identification through Concatenated Histograms of Local Appearance Features", IEEE Workshop on Applications of Computer Vision, 2007.

[18] H. Zhang, H. Mao, "Feature Selection for the Stored-grain Insects Based on PSO and SVM", Second International Workshop on Knowledge Discovery and Data Mining, 2009. pp.586-589, 23-25 Jan. 2009.

[19] M.D. Einheuser, el al., "Modeling the effects of conservation practices on stream health", Science of The Total Environment, Vol 435-436, 1 p.p. 380-391, 2012, ISSN 0048-9697.

[20] R.M.Sanchez, F.C.Espinosa, "Texel: a methodology and an integrated environment for developing automated systems for texture classification", IEEE International Joint Symposia on Intelligence and Systems, 1998. pp.382-388, 21-23 May 1998.

[21] Z.Z. Htike, "Can the future really be predicted?", Signal Processing: Algorithms, Architectures, Arrangements, and Applications (SPA), 2013 , pp.360-365, 26-28 Sept. 2013.

[22] Z.Z. Htike, "Multi-horizon ternary time series forecasting", Signal Processing: Algorithms, Architectures, Arrangements, and Applications (SPA), 2013, pp.337-342, 26-28 Sept. 2013.

[23] S.L. Win, et al., "Cancer Recurrence Prediction Using Machine Learning", International Journal of Computational Science and Information Technology (IJCSIT), 2014, Vol. 6, Issue 1, ISSN: 09753826.

[24] E.M. Azhari, et al., "Brain Tumor Detection and Localization in Magnetic Resonance Imaging", International Journal of Information Technology Convergence and services (IJITCS), 2014, Vol. 4, Issue 1, ISSN 2231-1939.

[25] N.A. Mohamad, et al., "Bacteria Identification from Microscopic Morphology Using Naïve Bayes", International Journal of Computer Science, Engineering and Information Technology (IJCSEIT), 2014, Vol. 4, Issue 1, ISSN 2231-3117.

[26] S.L. Win, et al., "Cancer Classification from DNA Microarray Gene Expression Data Using Averaged One-Dependence Estimators", International Journal on Cybernetics \& Informatics (IJCI), 2014, Vol. 3, Issue 1, ISSN: 2320-8430.

[27] E.M. Azhari, et al., "Tumor Detection in Medical Imaging: A Survey", International journal of Advanced Information technology (IJAIT), 2014, Vol. 4, Issue 1, ISSN 2231-1548.

[28] S.N.A. Hassan, et al., "Vision Based Entomology - How to Effectively Exploit Color and Shape Features”, Computer Science \& Engineering: An International Journal (CSEIJ), 2014, Vol. 4, Issue 1, ISSN: 2231-3583.

[29] N.A. Mohamad, et al., "Bacteria Identification from Microscopic Morphology: A Survey", International Journal on Soft Computing, Artificial Intelligence and Applications (IJSCAI), 2014, Vol. 3, Issue 1, ISSN: 2319-1015.

[30] S.L. Win, et al., "Gene Expression Mining for Predicting Survivability of Patients in EarlyStages of Lung Cancer”, 2014, Vol. 4, Issue 1, ISSN: 1839-9614. 